\title{
Influencia de las redes sociales en el trabajo de profesionales de la información especializados en salud. Caso de los Colegios Oficiales de Médicos de España
}

\author{
The influence of social networks on the work of information \\ professionals specializing in health. The Case of Spanish Official \\ Medical Associations
}

Dolores Rando Cueto. Programa de Doctorado Interuniversitario de Comunicación de las Universidades de Cádiz, Huelva, Málaga y Sevilla. España.

lrandocueto@uma.es

$[\mathrm{CV}] \odot \mathrm{G}$

Carlos de las Heras Pedrosa. Universidad de Málaga. España.

cheras@uma.es

$[\mathrm{CV}] \bigcirc \mathrm{G}$

Francisco Javier Paniagua Rojano. Universidad de Málaga. España.

fjpaniagua@uma.es

$[\underline{\mathrm{CV}}] \odot \mathrm{G}$

Cómo citar este artículo / Referencia normalizada

Rando Cueto, D., de las Heras Pedrosa, C., y Paniagua Rojano, F. J. (2021).. Influencia de las redes sociales en el trabajo de profesionales de la información especializados en salud. Caso de los Colegios Oficiales de Médicos de España. Revista Latina de Comunicación Social, 79, 113-133. https://www.doi.org/10.4185/RLCS-2021-1491

\section{RESUMEN}

Introducción. Los avances producidos en la libertad en el contenido informativo que fluye a través de las redes sociales digitales y una mayor participación de los usuarios en las mismas juegan un papel clave en la comunicación relacionada con la salud, en particular en la que generan los profesionales de la información especializados en el ámbito sanitario por el proceso de adaptación laboral que han contemplado y al que han de atender de acuerdo a la evolución de un escenario virtual cambiante. Este artículo se centra en la actividad vía social networks del más de Colegios Oficiales de Médicos (C.O.M.) de España, siendo el objetivo principal analizar de qué forma éstas influyen en la construcción de los mensajes que se emiten desde la institución a la población. Metodología. La investigación se basa en el desarrollo de una técnica metodológica cuantitativa a través de un cuestionario contestado por especialistas en información sanitaria de los colectivos 
referidos responsables de elaborar y gestionar la información emitida por parte de los C.O.M. valoran la comunicación que se intercambia en redes sociales digitales y consideran que Twitter $(81,3 \%)$ y Facebook (50\%) son las más relevantes de acuerdo con sus intereses, centrados en la promoción de la salud. Sobre las cuentas en redes sociales digitales utilizadas por los C.O.M. como fuentes informativas, son aquellas de entidades hospitalarias las que mayor credibilidad les merecen, aunque la mayoría no lo refleje en la construcción de su propio contenido. Conclusiones. Se pone en valor el potencial de las digital social networks, como canales de comunicación de gran utilidad para las organizaciones sanitarias para informar, establecer relaciones con diferentes públicos y escuchar las demandas de a los usuarios para retroalimentar el discurso en redes.

PALABRAS CLAVE: comunicación sanitaria; comunicación corporativa; redes sociales; hospitales; salud; Colegios Oficiales de Médicos.

\begin{abstract}
Introduction. The progress produced in the freedom in the informative content that flows through digital social networks and a greater participation of users in them play a key role in health-related communication, particularly in that generated by information professionals specialized in the health field due to the labor adaptation process that they have contemplated and which they have to attend to according to the evolution of a changing virtual scenario. This article focuses on the activity via social networks of the more than fifty Official Medical Colleges (C.O.M.) that exists in Spain. The main objective is analyze how these channels influence the construction of the messages that are emitted from the institution to the population. Methodology. The research is based on the development of a quantitative methodological technique through a questionnaire answered by specialists in health information from the referred groups. Results. Responsible for preparing and managing the information issued by the C.O.M. value the communication that is exchanged on digital social networks and consider that Twitter (81.3\%) and Facebook (50\%) are the most relevant according to their interests, focused on health promotion. About the digital social media accounts used by C.O.M. as information sources, it is those of hospital entities that deserve the most credibility, although most do not reflect it in the construction of their own content. Conclusions. The capacity of social networks is valued, as communication channels to inform, establish relationships with different stakeholders and listen to the demands of users to feed back the discourse on networks.
\end{abstract}

KEYWORDS: healthcare communication; corporate communication; social networks; hospitals; health; Medical Association.

\title{
CONTENIDO
}

1. Introducción. 2. Metodología. Objetivos e hipótesis. 3. Resultados. 4. Conclusiones. 5. Referencias.

\section{Introducción}

Hoy en día, la comunicación sanitaria juega un importante papel para los ciudadanos (Swan, 2009) y, por lo tanto, contribuye a la sostenibilidad social. La sociedad usa cada vez más internet para obtener información sobre salud, compartir experiencias relacionadas con procesos patológicos o encontrar personas con condiciones físicas o psicológicas similares (Swan, 2009; Van de Belt et al., 2013). Desde que las tecnologías de la información y la comunicación se utilizan en el campo de la salud, términos como e-paciente o e-salud se utilizan profusamente, lo que demuestra el rol cada vez más 
relevante que desempeñan los usuarios en la toma de decisiones sobre su bienestar (Tonia, 2014; Prasad, 2013).

Terrón (2007) ofrece su percepción desde un punto de vista antropológico. En su opinión, el interés por la información sanitaria en un país como España ha aumentado significativamente debido a la creciente necesidad de estilos de vida que conlleven un mayor bienestar social. Esta idea se refuerza por la oferta y la demanda de información: el interés en lo que se comunica crece a medida que se ofrece más información (Terrón, 2007; Vance, Howe y Dellavalle, 2009).

Internet se ha convertido en el "altavoz" más importante de las expectativas y demandas de los pacientes. Esto fomenta el surgimiento de asociaciones que apoyan los derechos de los pacientes de hacer oír su voz (Terrón, 2007, 24). Como resultado, esto hace que los pacientes se sientan más reforzados, al ser miembros de una plataforma de apoyo colectivo en la que pueden expresar sus necesidades y las de su entorno.

Las redes sociales digitales o digital social networks juegan un papel destacado en este sentido, con un aumento progresivo observado en términos de uso en el campo de la salud (Tonia, 2014; Ventola, 2014).

\subsection{Redes sociales digitales sanitarias. Ventajas}

Factores como la accesibilidad, la inmediatez o la capacidad para favorecer la comunicación bidireccional entre diferentes audiencias permiten una comunicación activa (Mangold y Faulds, 2009). Las entidades sanitarias, conscientes del potencial de las redes sociales, $y$ las utilizan para promover la interacción y la colaboración entre pacientes, familiares y profesionales (Sánchez et al., 2012).

Además de suscitar audiencias más dinámicas, los avances producidos en la libertad en el contenido informativo a través de las digital social networks (De las Heras-Pedrosa en al., 2019) en el campo de la salud, estos canales han provocado que el contenido sea más creíble al encontrarse los diferentes públicos en el mismo nivel de interacción. Se confiere un mayor poder comunicativo tanto a la ciudadanía, como a los profesionales sanitarios, cuando sus mensajes llegan a un mayor número de personas (Andersen, Medaglia y Enriksen, 2012; Bierglund-Andersen y Söderqvist, 2012).

Estos canales se han convertido en los instrumentos de comunicación para las corporaciones sanitarias, al facilitar la participación y colaboración con sus stakeholders y permitir, gracias a una comunicación bidireccional, el control de la calidad y la eficiencia de la institución (Griffis et al., 2014; Vance, Howe y Dellavalle, 2019), pero también para la educación de los ciudadanos con nuevos hábitos de vida saludable (Merchant, Elmer y Lurie, 2011; Kass-Hout y Alhinnarni, 2013). Además, es una herramienta clave para comunicar alertas sanitarias y la creación de redes de grupos de pacientes con las mismas patologías o de profesionales con fines de investigación (Roman, 2014; Bierglund-Andersen y Söderqvist, 2012).

Otra de las ventajas de las cuentas de entidades sanitarias en redes sociales digitales (Gomes y Coustasse, 2015; Ventola, 2014; Bernhardt, Alber y Gold, 2014) atiende a sus beneficios económicos, no solo porque su configuración sea gratuita, sino porque una mayor interacción entre profesionales sanitarios y pacientes puede evitar visitas presenciales a los centros sanitarios. Además, las digital social networks actúan también como herramientas para promocionar la actividad corporativa. Así, suponen un instrumento de comunicación multidimensional muy efectivo para 
asegurar la interacción con las partes interesadas, en este caso, profesionales de la salud y pacientes (Blázquez, Cantarero y Pascual, 2015).

Las cuentas de entidades sanitarias en digital social networks más populares son aquellas destinadas específicamente a personas que padecen alguna patología (Swan, 2009; Chou et al., 2009). De la Peña y Quintanilla $(2015,495)$ describen el papel que desempeñan para los ciudadanos como "una comunidad virtual donde pueden encontrar estimulación, obtener respuestas a preguntas específicas relacionadas con la salud y un lugar para compartir historias de éxito", y Koteyko, Hunt y Gunter (2015) agregan que esta herramienta tiene un gran potencial para promover iniciativas.

No obstante, otros sectores de la población también son receptores del contenido que circula en dichas redes convirtiéndose en stakeholders de estos canales de comunicación. De hecho, Swan (2009) sugiere que, en el campo de la salud, los profesionales destinados a atender a pacientes, investigadores y otros agentes involucrados en esta área podrían participar más activamente (Chou et al., 2009) en los mismos.

Desde el punto de vista del paciente, las redes sociales son el mejor instrumento para la interacción en tiempo real, permitiendo el intercambio de información y la participación, no solo como pacientes o usuarios, sino como grupos o asociaciones (Krowchuk, Lane y Twaddell, 2010). En el caso de los profesionales, se utilizan principalmente para difusión de resultados, investigación y creación de redes o la enseñanza, entre otros usos (Chretien y Kind, 2013).

La literatura científica relacionada con la comunicación sanitaria destaca la necesidad de los profesionales que la practican de adaptarse a los cambios provocados por la rápida invasión y evolución de las redes sociales digitales. Es necesario diseñar nuevas estrategias y desafíos para hacer frente a la aparición de un nuevo estilo de comunicación en red en tiempo real (Brandtzaeg et al., 2015). Los profesionales sanitarios pueden utilizar los nuevos escenarios de comunicación y su posición como fuentes autorizadas y creíbles para promover y defender la salud (Leask, Hooker y King, 2010).

Las digital social networks brindan a dichos especialistas en comunicación sanitaria información valiosa sobre experiencias de los pacientes, con la que pueden monitorear la reacción pública a los problemas de salud. También destaca el potencial de dicha información para el desarrollo de políticas de salud (Moorhead, et al., 2013). Un ejemplo son los blogs médicos, que son visitados frecuentemente por los medios de comunicación más importantes (Kovic, Lulic y Brunini, 2008).

\subsection{Redes sociales digitales sanitarias. Desventajas}

La democratización de la comunicaciónn a través de las redes sociales conlleva, en algunos casos, una falta de veracidad y control de la información (Bernhardt, Alber y Gold, 2014). Cualquiera puede provocar desasosiego en la sociedad con sus opiniones o comunicación de hechos no confirmados (Vance, Howe y Dellavalle, 2009), así como críticas perjudiciales o falsedades dirigidas a profesionales o instituciones sanitarios (Castilla, 2016). No se puede ignorar tanto los problemas legales, como la falta o violación de la privacidad del paciente, el daño a la imagen profesional, etc. (Chretien y Kind, 2013; Ventola, 2014; Bernhardt, Alber y Gold, 2014), así como la pérdida de privacidad o seguridad en la información compartida (Antheunis, Tates y Nieboer, 2013; Joseph Mattingly, 2015) y la falta de formación especializada tanto en salud como en el manejo de las redes sociales por parte de los profesionales de la comunicación (Rando-Cueto y De las Heras-Pedrosa, 2014; Rando-Cueto y De las Heras-Pedrosa, 2016). 
Para resolver problemas como estos, las entidades sanitarias y las organizaciones profesionales, como los Colegios Oficiales de Médicos, han desarrollado pautas de prevención y guías de buenas prácticas (Ventola, 2014) destinadas a proteger las instituciones, aquellos profesionales que atienden y asisten a los pacientes, los investigadores y otros agentes involucrados en este ámbito a nivel legal, clínico y organizacional (Lambert et al., 2012; Househ, 2013; Dizon et al., 2012; Childs y Martin, 2012).

Las asociaciones médicas, junto con las instituciones sanitarias juegan un papel crucial en el desarrollo de recomendaciones para el uso de las redes sociales. Por esta razón, el trabajo de los profesionales con un perfil de experto en comunicación y salud es esencial.

Leask, Hooker y King (2010) también destacan cómo el rol de los responsables de la comunicación sanitaria está perdiendo relevancia. Esto incurre en la desaparición de conocimientos técnicos básicos necesarios para transmitir la información sobre salud correctamente. Uno de los nuevos desafíos es desarrollar herramientas para verificar los contenidos, dado el riesgo de difundir información inexacta o falta generada por la velocidad cada vez mayor con la que emerge la información (Brantzaeg et al., 2015).

Rumores, noticias contradictorias, especulaciones, son características de los mensajes que circulan en redes sociales. En este sentido, no es concebible que el comunicador de salud dependa de fuentes que actúen como financiadores $\mathrm{u}$ y otros informantes con diferentes intereses, para comunicar a la sociedad de manera confiable y no ir en detrimento de su bienestar o calidad de vida (Gavilán e Iriberri, 2014). Por lo tanto, la ética y la responsabilidad en el trabajo, así como el compromiso con la sociedad son elementos clave del comunicador. De esta manera se ofrece una mejor atención a la población (Barrero y Palacios, 2015). Este trabajo se refleja cuando los intereses organizacionales se transfieren para reforzar el bienestar o la calidad de vida de los ciudadanos.

Aunque la veracidad de la información recae en el profesional de la comunicación, la determinación de la información correcta se complica en la actualidad debido, entre otros aspectos, a la gran cantidad de información generada por las redes sociales. Conocerlas y saber cómo usarlas para fines profesionales no es una tarea fácil y requiere nuevas formas de raciocinio. A pesar de esto, autores defienden este nuevo escenario debido a que el potencial comunicativo de las redes sociales no es desdeñable, "far from being negligible" (Kaplan y Haenlein, 2010, 67).

\subsection{Los C.O.M. en cuentas oficiales de redes sociales digitales}

Además de la verificación y credibilidad de la información difundida a través de las redes sociales, otros aspectos que los estudios analizados exponen son los efectos que éstas producen en la práctica profesional (Hermida, Lewis y Zamith, 2014); el uso que los profesionales hacen de ellos (Paulussen y Harder, 2014) y su importancia para los comunicadores o el peso que tienen con respecto a la información (Lariscy et al., 2009).

La modificación de las estructuras jerárquicas con respecto a la organización de la información por los espacios de conversación, conectividad y creación de comunidad que han provocado las digital social networks es uno de los cambios destacados por Hermida, Lewis y Zamith (2014) en el ejercicio de profesionales de la comunicación que trabajan el contenido de cuentas oficiales en redes sociales. Ya no existe un solo paradigma para la estructura de las noticias, pero hay muchas formas diferentes de desarrollar contenido. 
Lariscy et al. (2009), no sólo ponen el énfasis en la atención que las corporaciones deben tener en el contenido que se difunde a través de las social networks. Además, señalan que una de las tareas de los profesionales es monitorizar de cerca la información emitida por las entidades para las que trabajan y posiblemente involucrar a quienes originan ese contenido (Russell y Lamme, 2016), considerando que las redes sociales digitales contribuyen a la construcción de la agenda mediática. Incidiendo en la importancia de la supervisión de la información, las redes sociales digitales se consideran un instrumento alternativo para fomentar la comunicación entre las administraciones públicas y los diferentes stakeholders (Păun, 2009).

En concreto, en el sector de la comunicación vía digital social networks en el ámbito de la atención sanitaria especializada, las publicaciones científicas que se centran en la práctica profesional de los comunicadores especializados en salud son escasas (Rando-Cueto, Paniagua-Rojano y De las HerasPedrosa, 2016; Antheunis, Tates y Nieboer, 2013).

Los colegios profesionales juegan un papel primordial en la comunicación que promueven. El sector de los colegios profesionales en España es de gran importancia para la economía del país, principalmente debido al grado de empleo cualificado y su impacto en la calidad de los servicios de las empresas. Los servicios profesionales tienen un peso económico incuestionable, generan alrededor del 9\% del PIB y representan alrededor del 6\% del empleo total y el 30\% del empleo universitario. Por otro lado, la relevancia de los servicios profesionales radica en la protección de los derechos e intereses de los ciudadanos que los reciben.

A lo largo de diversas normativas sobre los colegios profesionales en España desde 1974, el sector ha sufrido diferentes transformaciones, pero mantiene la misma estructura que tenía originalmente. La ley define a los colegios profesionales como corporaciones de Derecho Público, protegidas por la Ley y reconocidas por el Estado, con su propia personalidad jurídica y plena capacidad para el cumplimiento de sus propósitos, lo que las empodera como entidades que representan y defienden la profesión que cada escuela tiene y señala el registro obligatorio para el ejercicio profesional regulado legalmente.

En el presente caso, los (-C.O.M.尹 españoles incentivan el trabajo científico de los médicos y sirven de puente entre dicho colectivo, los pacientes y la sociedad, en general. Pero, sobre todo, con sus comités deontológicos vigilan que las instituciones sanitarias se adhieran y cumplan las normas. Por esta razón, este estudio se centra en el grado de conocimiento que tales colegios profesionales tienen con respecto a las herramientas de comunicación actuales, entre las cuales las redes sociales juegan un papel influyente que afecta a la información que llega a los ciudadanos y a los pacientes, en particular. Por todo ello, se considera fundamental que existan profesionales especializados en comunicación y que estén a la vanguardia de los nuevos mecanismos y lenguajes comunicativos que se están desarrollando en la actualidad. Los C.O.M. deben continuar transmitiendo información fidedigna utilizando un lenguaje apropiado para toda la población.

\section{Metodología. Objetivos e hipótesis}

El principal objetivo de este estudio es analizar la influencia que sobre el ejercicio de los Colegios Oficiales de Médicos españoles tienen las redes sociales digitales. Se estudia cómo las emplean los profesionales responsables de la comunicación de estas entidades en la construcción de los mensajes que difunden, así como el valor que les merece lo que se publica en otras redes, que utilizan como fuente para la elaboración del contenido informativo. 
La investigación del uso que los profesionales de la comunicación que trabajan en los departamentos o agencias de comunicación de dichos Colegios hacen de las redes sociales se basa en las siguientes hipótesis:

H1. Existen profesionales especializados en comunicación en los C.O.M. que conocen perfectamente las nuevas tendencias comunicativas. Actualmente hay 52 Colegios Oficiales de Médicos en España con una estructura territorial y agrupadas en torno al denominado Consejo General de Colegios Oficiales de Médicos, cuya función es la representación exclusiva de estos a nivel nacional e internacional, así como la organización y defensa de la profesión médica.

Todas las delegaciones territoriales de colegios médicos tienen un departamento de comunicación organizado en el que trabajan de una a tres personas, ya sean especialistas en el campo o graduados en Relaciones Públicas y/o Periodismo. Además, estos se organizan en una red de comunicación de Colegios Oficiales de Médicos para intercambiar experiencias e incluso organizar un congreso anual para presentar y compartir sus iniciativas. Por ejemplo, en 2012 dedicaron esta reunión a las estrategias de redes sociales y también han trabajado en los últimos años en la lucha contra la difusión de noticias de salud falsas o comunicación de crisis.

H2. Los profesionales de la comunicación de los Colegios Oficiales de Médicos Españoles utilizan las digital social networks para su trabajo, tanto como fuente de información y verificación, como para establecer relaciones con otros profesionales y familiarizarse con los avances en su campo. En relación con la primera hipótesis y como resultado de la literatura científica previamente reportada, actualmente la participación de estos agentes en el escenario de las redes sociales tiene un gran impacto. A diferencia del comienzo de la década, cuando la presencia de los Colegios Oficiales de Médicos en España era insignificante, hoy su actividad es notable. Esto se nota en el aumento significativo tanto en el número de seguidores de sus redes sociales, como en el volumen de información que generan. La voz de los profesionales de estas entidades en las redes sociales adquiere especial relevancia para promover información precisa que preserva la calidad de vida y el bienestar de los ciudadanos.

H3. Para los profesionales de los Colegios Oficiales de Médicos, los contenidos compartidos en las cuentas oficiales de las redes sociales digitales de los centros sanitarios son aquellos que les merecen mayor credibilidad entre las que consultan. No obstante, rara vez reproducen estos mensajes en sus propias digital social networks. Los contenidos compartidos en las cuentas oficiales de los centros hospitalarios no se consideran valiosos entre los profesionales de los Colegios Oficiales de Médicos, ya que no abordan las demandas del público. A pesar del uso de las redes sociales llevadas a cabo por los profesionales de la comunicación de las entidades mencionadas, como se indica en la segunda hipótesis, en el caso de las cuentas corporativas de los hospitales, carecen de impacto con respecto al contenido de la información que publican los colectivos médicos. Esta premisa también implica una falta de interacción entre los centros hospitalarios y los colegios médicos a través de las digital social networks.

Un mayor volumen de información dirigida específicamente a cubrir las necesidades de información o comunicación de audiencias, como los profesionales de los Colegios Médicos, derivaría en una audiencia más amplia y en la difusión más efectiva de sus contenidos, algo que sería especialmente útil para la sociedad en casos de alertas de salud, por ejemplo, o situaciones que requieran una reacción rápida de los ciudadanos, con respecto a cuestiones sanitarias. 
Con la intención de profundizar en el uso que los profesionales de la comunicación especializados en salud realizan de las redes sociales se realizó una revisión sistemática de la literatura científica internacional publicada en la última década.

Al mismo tiempo, el estudio se apoya en una encuesta, "una de las técnicas más utilizadas en investigación social" (Ruiz-Olabuénaga, 2012, 72), que consiste en la realización de preguntas organizadas de forma sistemática, las cuales pueden ser abiertas o cerradas, con respuestas únicas o múltiples y que ofrece datos e información de forma homogénea. Esto permite, según Igartua (2006, 231), "la obtención de datos sobre aspectos objetivos (hechos) y subjetivos (opiniones, actividades) basada en la información (oral o escrita) proporcionada por el sujeto" (Álvarez González, y Cea D’Ancona 1997; Neuendorf, 2017).

En este sentido, Malhotra (2006, 115-168) considera que las encuestas son cuestionarios planteados a un gran número de personas. Para ello se utiliza un cuestionario prediseñado de antemano, con el objetivo de obtener información específica. Este tipo de metodología analiza actitudes que no se pueden observar de forma directa, por lo que facilita descubrimientos descriptivos. Macionis y Plummer (2012) definen las encuestas como un conjunto de preguntas redactadas por un investigador y enviadas a sujetos para que respondan a estas cuestiones (Piñuel, 2002; Riffe, Lacy y Fico, 2005).

El cuestionario diseñado para este estudio se centró en rutinas profesionales, uso de redes sociales: tiempo invertido, frecuencia de empleo, motivos, cuentas preferidas, y, por último, valorar aspectos sobre la importancia de estos canales de comunicación en el trabajo, credibilidad, contenidos y necesidades informativas.

El cuestionario constó de siete bloques, de los que se trató de identificar de qué manera las redes sociales digitales influyen en la labor desarrollada por los profesionales de la información en salud de los Colegios Oficiales de Médicos de España. En la primera parte, se preguntó sobre la edad, el grado y algunos datos sobre funciones y tareas, así como la antigüedad laboral, basados en una herramienta validada por los estudios de European Monitor Communication (Zerfass et al., 2018).

A partir de aquí, se incluyeron diferentes preguntas sobre el uso de las redes sociales por parte de los responsables de comunicación en su trabajo diario. Hubo seis preguntas de respuesta cerrada y tres casos de respuestas múltiples (es decir, con más de una respuesta posible).

Si bien, primeramente, el interés era conocer la frecuencia (número de horas al día) con que los profesionales acudían a los referidos canales de comunicación para realizar su trabajo, luego fueron consultados sobre las principales razones por las que usan las redes profesionales en su rutina profesional: como fuente de información, para interactuar con periodistas especializados, como un canal de comunicación más, para establecer relaciones profesionales, para buscar historias relacionadas con su temática de trabajo o para contrastar información.

En tercer lugar, se preguntó a los responsables de comunicación sobre la importancia que les dan a las redes sociales en el sector -ya sea relevante o irrelevante-. Posteriormente, se quiso conocer sus opiniones sobre cómo éstas contribuyen a la construcción de la agenda-setting en el sector sanitario. En este punto, se identificaron las demandas de información en las redes sociales de los responsables de la comunicación: información de servicios, investigación, información general sobre salud, información sobre la actividad hospitalaria o información sobre promoción de la salud. 
Finalmente, se realizó una pregunta con respecto a los perfiles de los públicos destinatarios de la información que los colegios profesionales comparten en social networks (redes de salud o grupos de hospitales, pacientes y familiares, personal del hospital y ciudadanos) y la importancia de la comunicación en redes sociales con cada uno de ellos.

El cuestionario referido se remitió a los todos los los 52 C.O.M. de España. Se dirigieron al responsable de comunicación y en el caso de que este no figurase en la información pública del Colegio al Decano como máximo representante. Los datos se recogieron durante los meses de noviembre de 2017 y enero del 2018, a través de una base de datos sobre la que se llevó a cabo la interpretación de resultados. Es importante destacar, que no todos los C.O.M cuentan con un departamento de comunicación, y algunos tienen este servicio contratado fuera, por lo que se puede afirmar que el número de encuestados, 38, (el 73’07\%) mantiene el nivel de confianza estadística y el margen de error recomendado para este estudio.

De forma más concreta, los encuestados respondieron sobre cuestiones relacionadas con el empleo que hacen de las redes sociales como parte de su trabajo (objetivos que persiguen al consultarlas o relevancia de las mismas en la construcción de su agenda informativa) y la consideración que les merecen como fuente informativa y en su productividad laboral. Además, se les pidió que sugirieran mejoras que consideraban relevantes en la comunicación vía social networks en aras a optimizar su labor profesional.

Con las respuestas obtenidas se ha trazado una radiografía de la influencia que las redes sociales sanitarias, ejercen sobre el ejercicio de los profesionales de la comunicación en la producción de contenido o en la previsión de su agenda-setting.

\section{Resultados}

Los resultados analizados en el estudio corroboran y completan las hipótesis planteadas al comienzo de la investigación. Con respecto a la primera de las hipótesis indicadas, los resultados proporcionan un perfil claro del especialista en salud que trabaja en comunicación de instituciones o colegios profesionales, el cual, tanto por sus años de experiencia en el sector de la comunicación sanitaria, como por su actividad en tas redes sociales digitales validan su amplio conocimiento en el campo. Esta persona tiene más de 30 años y más de 5 años de experiencia profesional; trabaja principalmente en colegios profesionales en el sector de la salud; y es indiscutiblemente activa en social networks.

Más de la mitad de la muestra, $57.1 \%$, tiene entre 30 y 39 años, mientras que el resto tiene más de 40 (21.4\% tiene entre 40 y 49 años y el mismo porcentaje, entre 50 y 65 años). Todos ellos formaban parte de la población en edad laboral cuando aparecieron las redes sociales, según el mapeo del nacimiento con la evolución de las mismas que Boyd y Ellison hicieron en 2008 (Boyd y Ellison, 2007; Colás-Bravo, González-Ramírez y De-Pablos-Pons, 2013).

La cantidad de años de experiencia profesional también merece atención. Más de la mitad de los profesionales ha trabajado durante más de diez años $(57,1 \%$ y el resto ha trabajado entre cinco y nueve años (42.9\%). Esto es significativo si consideramos que la especialización en el sector de la salud, que desde los años 90 se ha visto afectado por una "superabundancia de información" (Mercado, 2013) implica años de experiencia profesional para proporcionar información de calidad.

En términos de usabilidad, el empleo recurrente de las social networks como instrumento de trabajo expuesto en la literatura científica mencionada se ratifica en los resultados obtenidos en el 
cuestionario. El hecho de que todos los encuestados empleen las redes sociales en su actividad profesional podría tener un efecto sobre cómo los gestores de las instituciones sanitarias perciben estos canales corporativos, ya que, en algún momento durante el día, podrían estar expuestos a los medios de comunicación. (ver gráfico 1)

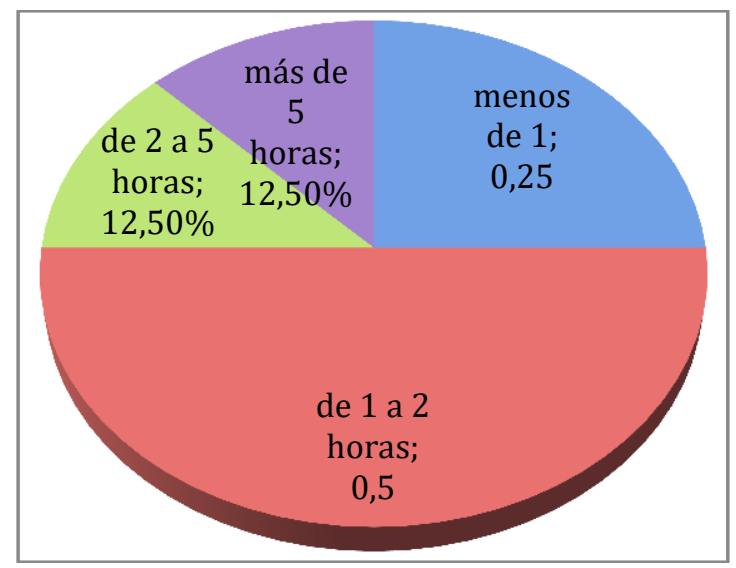

Gráfico 1: Uso de las redes sociales al día.

Fuente: Elaboración propia.

En particular, de las redes sociales empleadas, los profesionales especializados en salud en el ámbito nacional acuden con mayor asiduidad a Twitter (87.5\%) y Facebook (81.3\%).

En cuanto al uso de las digital social networks realizado por los profesionales encuestados, los resultados muestran una casuística mayor que las posibilidades planteadas en la segunda hipótesis del estudio. A las razones expuestas anteriormente, el uso de las redes sociales como fuente informativa y de verificación y como forma de establecer relaciones con otros profesionales para aprender sobre los avances en su campo, se suman otros factores. Así, los profesionales también destacan la monitorización de la competencia y localización de testimonios relacionados con la salud.

En el caso de las redes sociales como fuente de información, la diferencia entre el porcentaje de usuarios de Twitter y de Facebook aumenta, por lo que Twitter se posiciona como la red social elegida en primer lugar por los profesionales especializados como fuente informativa (con un $81.3 \%$ de las respuestas obtenidas, frente a un $50 \%$ otorgado a Facebook).

En cualquier caso, las digital social networks se han convertido en una fuente de información relevante (el $25 \%$ de los encuestados lo considera de esta manera) o muy importante para la mayoría de los profesionales (un 62.5\%).

De hecho, el $87.5 \%$ de los encuestados emplea las redes sociales "como fuente informativa", como uno de los principales objetivos de su interacción con éstas. Un 56.3\% destaca como propósito de uso el de "conocer lo que ocurre en su ámbito de trabajo". En orden de importancia, le sigue el interés por: "actuar como fuente informativa del medio para el que trabaja"; "utilizar las redes sociales como protagonistas del propio contenido informativo"; "establecer relaciones profesionales"; "monitorizar la competencia"; "actuar como fuente informativa como periodista especializado"; "contrastar información"; y "buscar historias" (ver gráfico 2). 


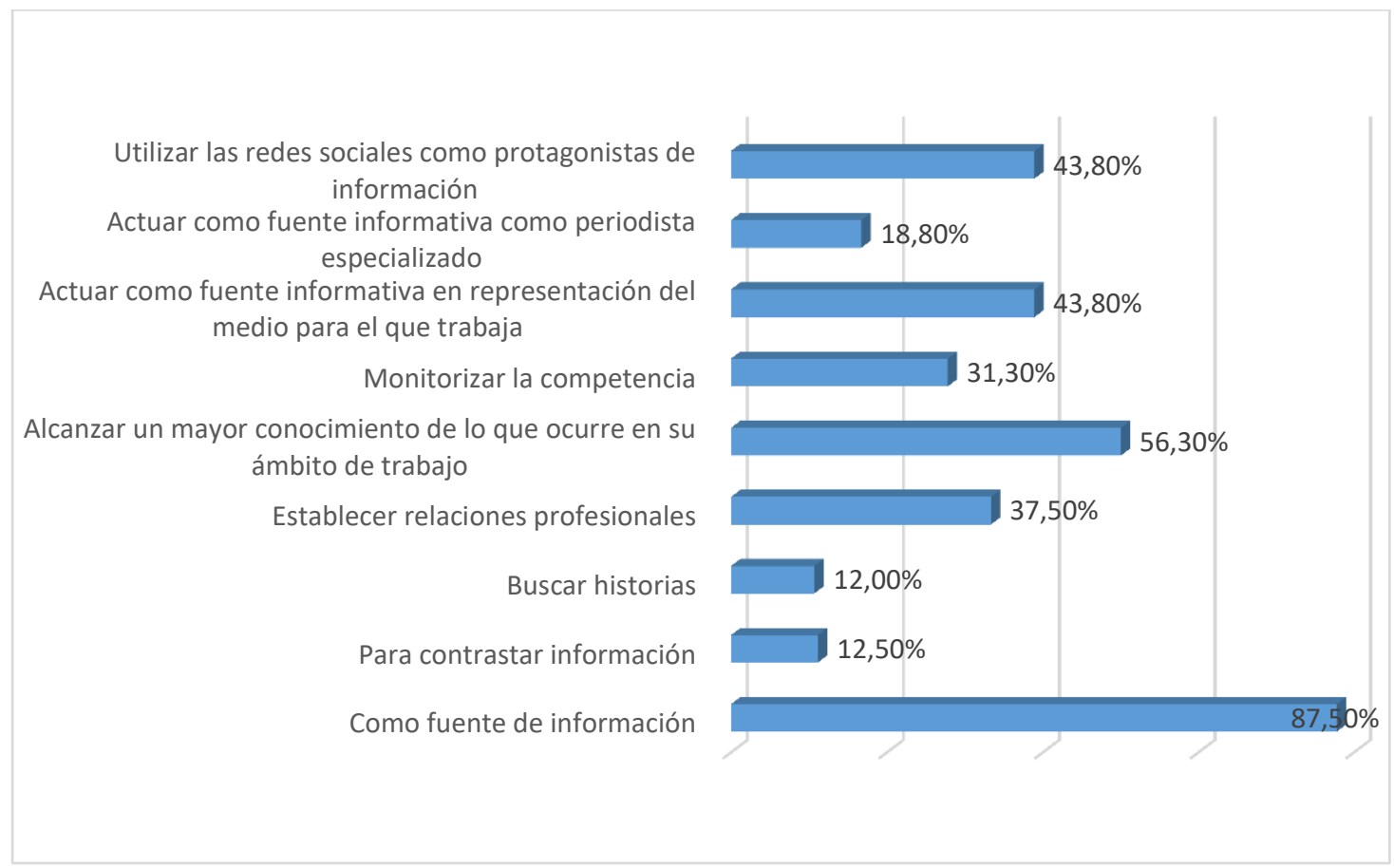

Gráfico 2: Principales objetivos en el uso de las RR.SS.

Fuente: Elaboración propia

Es significativo que, a pesar de la importancia que a los encuestados les merece la comunicación sanitaria vía redes sociales digitales, estos reconozcan que este contenido tiene no tiene el valor suficiente para formar parte de ła su agenda. Como se indicó en la tercera hipótesis, lo que se publica a través de los canales mencionados no satisface la demanda de información de salud manifestada por la sociedad.

Acerca de la autoría de las social networks, todas las personas consultadas que visitan cuentas relacionadas con el sector salud acceden a las redes sociales corporativas de los hospitales, calificando su contenido como "importante" seguido de "muy importante", "poco importante" y "neutral" (Ver gráfico 2). Ninguno de los encuestados califica como "nada importante" el contenido encontrado en las redes sociales hospitalarias.

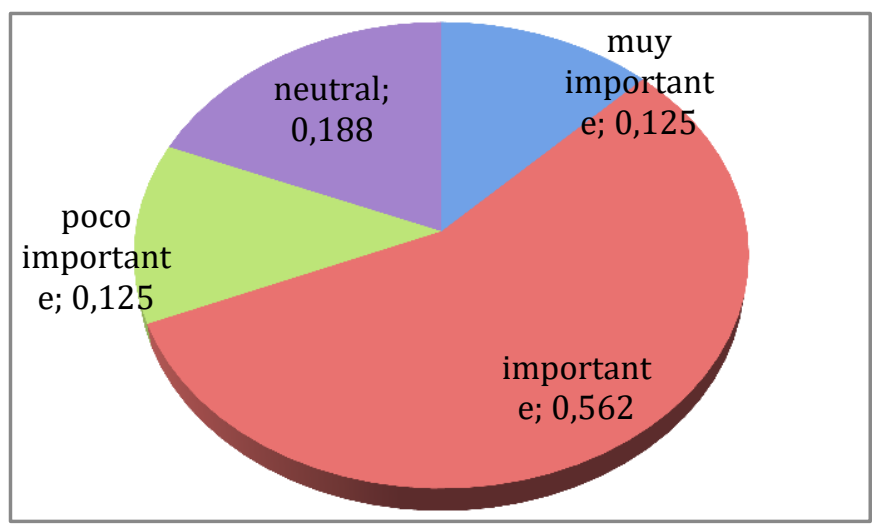

Gráfico 3: Importancia de las RR.SS. relacionadas con el sector salud.

Fuente: Elaboración propia. 
Es notable el seguimiento de redes sociales sanitarias por parte de profesionales especializados en salud cuya gestión no compete a una institución, sino que sus gestores son profesionales sanitarios e investigadores (un 56.3\%). El 31.3\% de los encuestados menciona a colectivos de pacientes que también comunican a través de dichos canales.

Sin embargo, las cuentas en redes procedentes de las corporaciones sanitarias son las que mayor credibilidad merecen con el $87.5 \%$. En términos de credibilidad, a este porcentaje le siguen las cuentas de profesionales sanitarios e investigadores del sector salud con el 31.3\%. Si los sectores público y privado se consideran por separado, las cuentas del sector sanitario público disfrutan de mayor credibilidad que las del ámbito privado: un $18.8 \%$ frente a un 12 ' $5 \%$, respectivamente; porcentaje este último igual que al otorgado a las cuentas de colectivos de pacientes (12.5\%). Las cuentas de ciudadanos que se expresan sobre salud y que no son miembros de asociaciones no gozan de credibilidad en el estudio.

El argumento que las personas consultadas dan por sus respuestas gira en torno al rigor científico $(18.75 \%)$, que considera mayor en el caso de las instituciones sanitarias. La fiabilidad de la información que se vierte en este tipo de redes sociales vendría respaldada por la institución a la que representa y la profesionalidad de sus especialistas.

Sobre la relación entre la información ofrecida por los hospitales y cómo esta es percibida por los profesionales de los C.O.M. españoles, analizada para respaldar la tercera hipótesis, se extraen diferentes resultados. Uno de los comentarios hace referencia a la parcialidad del contenido de las informaciones ofrecidas por parte del sector público. Así, los hospitales públicos estarían publicando contenido sesgado en sus cuentas al "no exponer abiertamente asuntos controvertidos" y remitirse solo a "datos de relevancia política" o presentar temas "que puedan tener un rédito político".

No obstante, al valorar del 1 al 10 la credibilidad de las cuentas de los centros hospitalarios, siendo 10 la mayor calificación, más de la mitad de los profesionales, el 68.75\%, aprueba a las redes sociales hospitalarias en lo que a fiabilidad se refiere, si bien el resto les concede un suspenso o no las sigue. De acuerdo con los resultados expuestos, comentarios que aluden a la falta de esta credibilidad hacen referencia al sesgo que pueden tener los contenidos que reflejan una visión parcial de la realidad.

Hay que destacar cómo, a pesar de que un $18.8 \%$ de los encuestados considera que las redes sociales digitales entorpecen su trabajo porque en las mismas se vierte demasiada información y, en ocasiones, ello produce intoxicación en el contenido, un $75 \%$ de los encuestados piensa que facilitan su trabajo y les conducen a otras fuentes informativas.

A pesar de este dato, por lo que respecta a la construcciónn de la agenda-setting, una amplia mayoría $(93.3 \%)$ expone que el contenido de las redes sociales hospitalarias conforma menos de la mitad de la información que elabora diariamente. Este porcentaje es muy revelador a la hora de describir cómo los medios de comunicación utilizan el contenido que se publica en éstas (ver gráfico 4). 


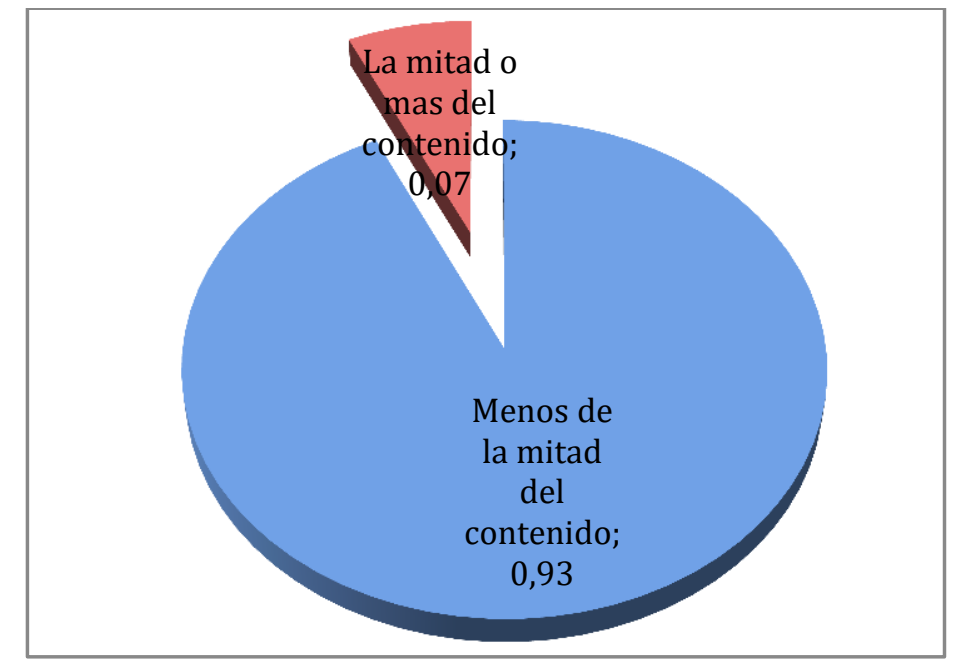

Gráfico 4: Contribución de las RR.SS. hospitalarias a la agenda setting.

Fuente: Elaboración propia.

En sus respuestas, los profesionales sugieren cambios generales en el contenido de las redes sociales, lo que se vincula con el argumento presentado en la tercera hipótesis.

El $66.7 \%$ de los participantes en la encuesta considera que modificaría las redes sociales hospitalarias tal y como las encuentran hoy y, de este porcentaje, el $88.9 \%$, realizaría cambios con relación a la información que se difunde en las mismas. En la mayoría de los casos, el contenido publicado por las redes sociales del hospital no se ajusta a los objetivos profesionales de los responsables de los departamentos de comunicación.

Menos de la mitad ve cumplidos los fines que persigue cuando consulta una red social hospitalaria (46.7\%). Los contenidos que más les interesan son los relacionados con la salud en general, seguidos de aquellas sobre promociónn sanitaria, investigación, información de servicio y, por último, información sobre actividad del hospital (ver gráfico 5).

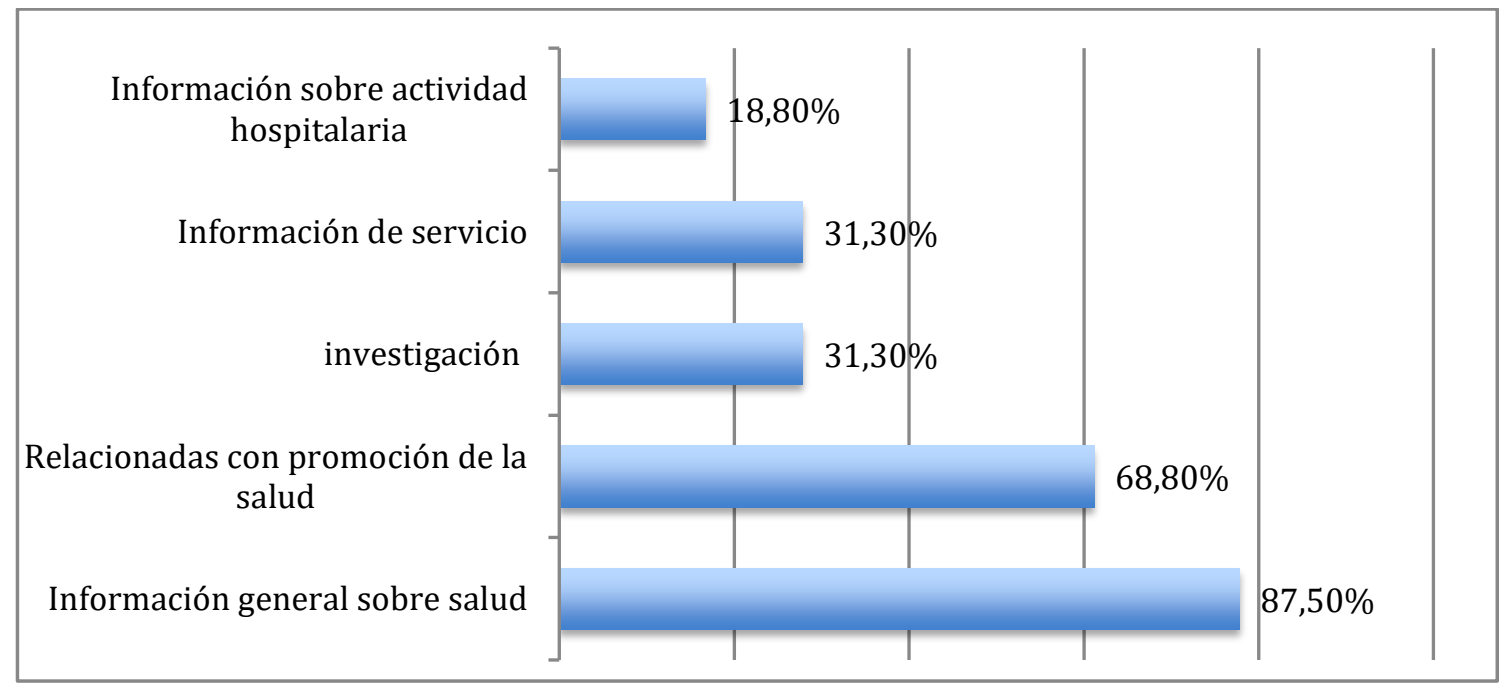

Gráfico 5: Cumplimiento de objetivo tras la consulta en RR.SS.

Fuente: Elaboración propia. 
A su vez, los actores que más interés suscitan a los profesionales de comunicación, por orden de importancia, son los propios trabajadores del hospital, seguidos del mismo hospital como institución, los pacientes y familiares, los ciudadanos en general $\mathrm{y}$, por último, la red sanitaria o grupo hospitalario (ver gráfico 5).

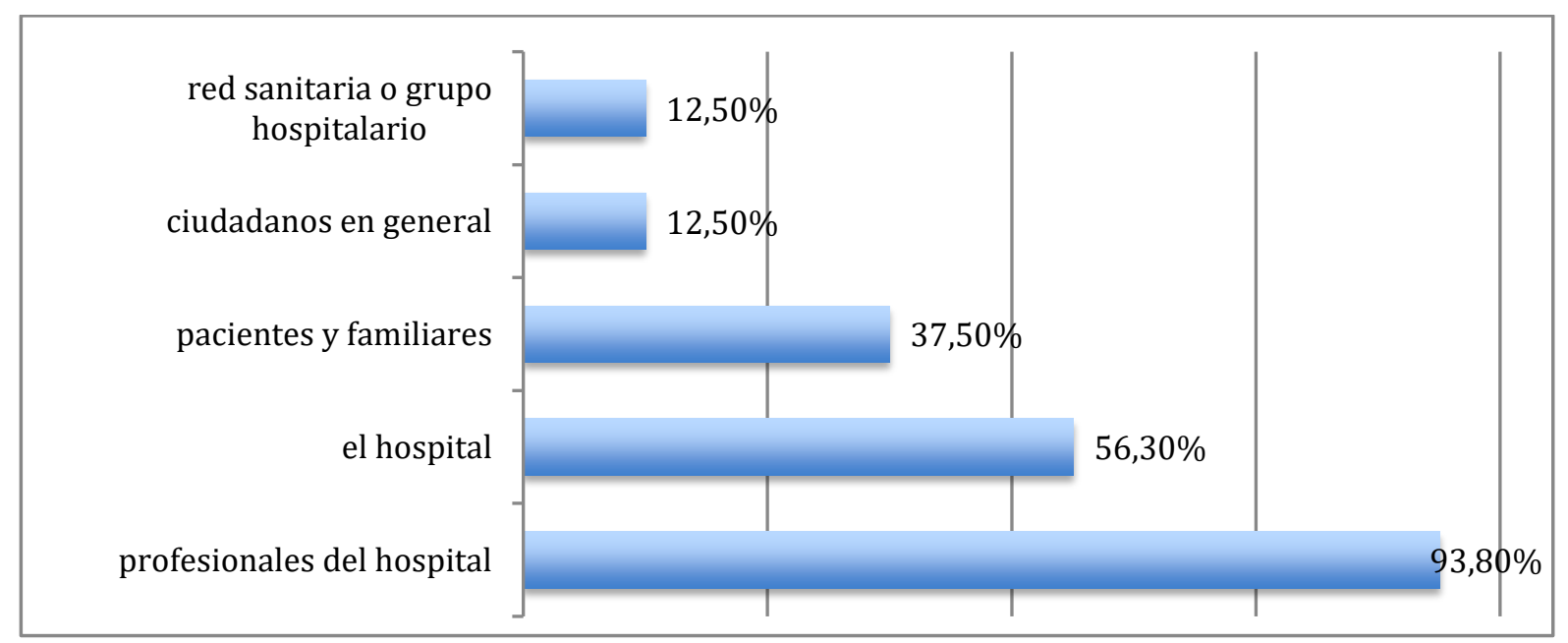

Gráfico 6: Importancia de los públicos primarios.

Fuente: Elaboración propia.

Otros aspectos que modificarían los profesionales de comunicación son aquellos relacionados con el aspecto o la capacidad comunicativa entre las partes implicadas en la misma. No obstante, en ninguna de las respuestas obtenidas se han plasmado consideraciones de mejoras concretas.

En sus respuestas, los profesionales de los medios especializados en salud mencionan cómo las instituciones evitan la gestión de la comunicación a través de las redes sociales en caso de crisis sanitarias. Es ampliamente reconocido que las social networks ofrecen una circulación inmediata de mensajes, lo que las válida para dicha comunicación profesional.

La rapidez con la que la información se puede difundir a través de las redes sociales en situaciones de emergencia, catástrofes y eventos socialmente críticos o de calado social, que supongan una alarma para la salud pública, hace a los referidos canales esenciales para los profesionales. Este grupo tiene la capacidad y el poder de convertirse en altavoces de los centros hospitalarios en su papel de fuentes oficiales en materia de salud, utilizando las redes sociales corporativas en momentos en los que la ciudadanía demanda un suministro continuo de noticias.

\section{Conclusiones}

Se puede concluir que la comunicación es esencial para los Colegios Oficiales de Médicos (C.O.M.). Como hemos visto, los C.O.M. cuentan con especialistas en comunicación con amplios conocimientos en el trabajo que desarrollan como parte de su personal. Así se han expresado los participantes en el estudio, representando el $69.2 \%$ de estas entidades en el ámbito español. Ello confirma la primera hipótesis indicada.

La segunda hipótesis también se confirma pues todos los profesionales de la comunicación que han contestado utilizan las redes sociales como herramienta de trabajo. Twitter y Facebook son las más 
usadas, si bien es cierto que, como fuente de información, la primera es más popular $(81.3 \%)$ que la segunda $(50 \%)$.

Con respecto a la hipótesis tercera al evaluar la comunicación que otras entidades sanitarias, como hospitales, desarrollan a través de sus redes sociales corporativas, ha sido productivo analizar cómo el contenido vertido a través de las mismas influye en la práctica diaria de los profesionales especializados en salud que trabajan en los departamentos de comunicación de los C.O.M. La aportación de estos como grupos de interés de los centros de atención sanitaria especializada, intermediarios entre institución y sociedad, y a su vez, parte implicada en la construcción de la realidad, ha servido para entrever fortalezas y debilidades de las cuentas oficiales de centros hospitalarios en redes sociales digitales.

Una escucha activa por parte de las instituciones sanitarias, en concreto de los gestores de la comunicación hospitalaria sobre las necesidades que encuentran los profesionales de los colegios profesionales del sector sanitario a nivel nacional se plantea como reto para acercar y conseguir un mayor provecho a las relaciones profesionales entre ambas partes.

Los encuestados demandan cambios en las social networks de los hospitales, entre los que destaca: la oferta de contenidos no sesgados de acuerdo con intereses corporativos; más información sobre salud, en general, y sobre promoción de la misma que pueda transmitirse a la ciudadanía; así como un mayor protagonismo de los profesionales que trabajan en el hospital, en lugar de que sea la entidad quien se dirija a la sociedad como institución.

Los participantes en el estudio consideran que las redes sociales son relevantes, es decir, conocen su potencial y valoran el volumen de información que encuentran en éstas, aunque su contenido conforme una mínima parte de la agenda-setting que gestionan. En este sentido, una mayoría considera a las redes sociales como facilitadoras de su tarea, como una herramienta más a utilizar en su ejercicio profesional, pero son pocos los que las incluyen abiertamente como fuente en sus contenidos informativos.

La necesaria adaptación de las redes sociales institucionales a los objetivos de los profesionales especializados en salud revertiría los porcentajes señalados al respecto.

La credibilidad de las redes sociales corporativas, basada en la veracidad de la información que publican es muy valorada por los profesionales de los C.O.M. No obstante, es significativo que un porcentaje de los encuestados, aun siendo menos de la mitad, encuentre que no es satisfactorio el contenido referido, por no ser completo y ofrecer una información de acuerdo con los intereses de la entidad hospitalaria.

Para que los profesionales participantes en la encuesta cambien su visión de las referidas fuentes informativas, la comunicación entablada en las cuentas de centros sanitarios, como los hospitalarios, en redes sociales digitales debe basarse en un contenido veraz, sin sesgos y transmitido por agentes que desempeñen un papel central en el contenido informativo. El eje principal no puede ser siempre el de dar a conocer la actividad del centro hospitalario ni la venta de un producto o servicio, sino que el hilo conductor de la información ha de estar enfocado a la promoción de la salud y del bienestar ciudadano.

Los responsables de establecer la agenda setting sanitaria han expresado que en tales circunstancias serían más permeables a la información procedente de las redes sociales hospitalarias, que desempeñarían un papel más destacado como fuentes informativas relevantes. 


\section{Referencias}

Álvarez González, R., \& Cea D’Ancona, M.A. (1997). Metodología Cuantitativa. Estrategias y técnicas de investigación social. Reis, (80), 240-242. http://dx.doi.org/10.2307/40183928

Andersen, K. N.; Medaglia, R.; Enriksen, H. Z. (2012). Social media in public health care: Impact domain propositions. Gov. Inform. Q., 29, 462-469. http://dx.doi.org/10.1016/j.giq.2012.07.004

Antheunis, M.L., Tates, K.; Nieboer, T.E. (2013). Patients' and health professionals' use of social media in health care: Motives, barriers and expectations. Patient. Educ. Couns., 92, 426-431. http://dx.doi.org/10.1016/j.pec.2013.06.020

Barrero, A. E., \& Palacios, J. A. (2015). Reflexiones sobre el papel del comunicador social y competencias del comunicador en las organizaciones. Poliantea. doi:10.15765/plnt.v11i20.659

Bernhardt, J.M.; Alber, J.; Gold, R.S. (2014). A social media primer for professionals: digital do's and don'ts. Health Promotion Practice, $15, \quad 168-172$. http://dx.doi.org/10.1177/1524839913517235

Bjerglund-Andersen, N.; Söderqvist, T. (2012). Social Media and Public Health Research. University of Copenhagen, Faculty of Science: Copenhagen. http://www.bjerglund.files.wordpress.com/2012/11/final-social-media-and-public-healthresearch1.pdf

Blázquez Fernández, C.; Cantarero Prieto, D.; Pascual Sáez, M. (2015). Promoting the use of health information and communication technologies in Spain: a new approach based on the ICT-H. Revista ICONO14 Revista científica de Comunicación y Tecnologías emergentes, 13, 238-259. http://dx.doi.org/10.7195/ri14.v13i2.860

Boyd, D.; Ellison, N. (2007). Social Network Sites: Definition, History, and Scholarship. J. ComputMediat Comm., 13, 210-230. http://dx.doi.org/10.1111/j.1083-6101.2007.00393.x

Brandtzaeg, P. B., Lüders, M., Spangenberg, J., Rath-Wiggins, L. \& Følstad, A. (2015). Emerging Journalistic Verification Practices Concerning Social Media. Journalism Practice, 10(3), 323-342. doi:10.1080/17512786.2015.1020331.

Castilla, G. (2016). La Comunicación en salud desde el punto de vista de una sociedad médica. $\begin{array}{llllll}\text { Revista Española de Comunicación } & y & \text { Salud, } & 7, & 129-132 .\end{array}$ http://dx.doi.org/10.20318/recs.2016.3131

Colás-Bravo, P., González-Ramírez, T. \& de Pablos-Pons, J. (2013). Young People and Social Networks: Motivations and Preferred Uses. Comunicar, 20(40), 15-23. http://dx.doi.org/10.3916/c40-2013-02-01

Chretien, K.C.; Kind, T. (2013). Social Media and Clinical Care. Circulation, 127, 1413-1421. http://dx.doi.org/10.1161/circulationaha.112.128017 
Childs, L.M.; Martin, C. Y. (2012). Social media profiles: striking the right balance. Am. J. HealthSyst Ph., 69, 2044-2050. https://doi.org /10.2146/ajhp120115

Chou, W. S.; Hunt, Y. M.; Beckjord, E. B.; Moser, R. P.; Hesse, B. W. (2009). Social media use in the United States: implications for health communication. J. Med. Internet. Res., 11, e48. http://dx.doi.org/10.2196/jmir.1249

De la Peña, A. \& Quintanilla, C. (2015). Share, like and achieve: the power of Facebook to reach health-related goals. International Journal of Consumer Studies, 39(5), 495-505. http://dx.doi.org/10.1111/ijcs.12224

De las Heras Pedrosa, C; Jambrino-Maldonado, C.; Iglesias-Sánchez, P.P.; Lugo-Ocando, J. (2019). Importancia de las relaciones con los públicos en la reputación en un destino turístico inteligente. Propuesta de un modelo sostenible. Rev. Int. Relat. Public., 9, 117-138. http://dx.doi.org/10.5783/rirp-17-2019-07-117-138

Dizon, D. S.; Graham, D.; Thompson, M. A.; Johnson, L. J.; Johnston, C.; Fisch, M. J.; Miller, R. (2012). Practical guidance: the use of social media in oncology practice. J. Oncol. Pract., 8, e114e124. https://doi.org /10.1200/JOP.2012.000610

Gavilan, E. \& Iriberri, A (2014). Medios de Comunicación como agentes que facilitan la medicalización de la vida: el ejemplo de la andropausia. Revista de Comunicación y Salud, 4, 4967

Gomes, C.; Coustasse, A. (2015). Tweeting and Treating: How Hospitals Use Twitter to Improve Care. The Health Care Manager, 34, 203-214. http://dx.doi.org/10.1097/hcm.0000000000000063

Griffis, H.M.; Kilaru, AS, Werner, R.M.; Asch, D.A.; Hershey, J.C.; Hill, S.; Ha, Y.P.; Sellers, A.; Mahoney, K.; Merchant, R.M. (2014). Use of Social Media Across US Hospitals: Descriptive Analysis of Adoption and Utilization. J. Med. Internet. Res., 16, e264. http://dx.doi.org/10.2196/jmir.3758

Hermida, A., Lewis, S. C., \& Zamith, R. (2014). Sourcing the Arab spring: A case study of Andy Carvin's sources on twitter during the Tunisian and Egyptian revolutions. Journal of ComputerMediated Communication, 19(3), 479-499. http://dx.doi.org/10.1111/jcc4.12074

Househ, M. (2013). The use of social media in healthcare: organizational, clinical, and patient perspectives. St. Hea.l T., 183, 244-248. https://doi.org/10.3233/978-1-61499-203-5-244\}

Igartua Perosanz, J.J. (2006). Métodos cuantitativos de investigación en comunicación. Barcelona: Editorial Bosch.

Joseph Mattingly, T. (2015). Innovative patient care practices using social media. Journal of the American Pharmacists Association, 55(3), 288-293. http://dx.doi.org/10.1331/japha.2015.14171

Kaplan, A. M. \& Haenlein, M. (2010). Users of the world, unite! The challenges and opportunities of Social Media. Business Horizons, 53(1), 59-68. http://dx.doi.org/10.1016/j.bushor.2009.09.003 
Kass-Hout, T.; Alhinnami, H. /2013). Social media in public health. Brit. Med. Bull., 108, 5-24. http://dx.doi.org/10.1093/bmb/ldt028

Koteyko, N., Hunt, D. \& Gunter, B. (2015). Expectations in the field of the Internet and health: an analysis of claims about social networking sites in clinical literature. Sociology of Health \& Illness, 37(3), 468-484. http://dx.doi.org/10.1111/1467-9566.12203

Kovic, I., Lulic, I. \& Brumini, G. (2008). Examining the Medical Blogosphere: An Online Survey of Medical Bloggers. Journal of Medical Internet Research, 10(3), e28. http://dx.doi.org/10.2196/jmir.1118

Krowchuk, H.V.; Lane, S.H.; Twaddell, J.W. (2010). Should Social Media be Used to Communicate With Patients? Mcn-Am. J. Matern-Chil., $\quad 35, \quad 6-7$. http://dx.doi.org/10.1097/01.nmc.0000366802.88540.54

Lambert, K.M.; Barry, P.; Stokes, G. (2012). Risk management and legal issues with the use of social media in the healthcare setting. J. Healthc. Risk Manag., 31, 41-47. http://dx.doi.org/10.1002/jhrm.20103

Lariscy, R. W., Avery, E. J., Sweetser, K. D., \& Howes, P. (2009). An examination of the role of online social media in journalists' source mix. Public Relations Review, 314-316. https://doi.org/10.1016/j.pubrev.2009.05.008

Leask, J., Hooker, C. \& King, C. (2010). Media coverage of health issues and how to work more effectively with journalists: a qualitative study. BMC Public Health, 10(1). http://dx.doi.org/10.1186/1471-2458-10-535

López Villafranca, P. \& Castillo Esparcia, A. (2017). Comunicación y enfermedades raras. La gestión de la comunicación de las organizaciones de pacientes. Portugal: LabCom.

Macionis, J. \& Plummer, K. (2012). Sociology: A global Introduction. Harlow: Pearson.

Malhotra, N. K. Questionnaire Design and Scale Development (2006). In The Handbook of Marketing Research: Uses, Misuses, and Future Advances, Grover, R., Vriens, M., Eds.; Sage Publications Ltd: London. U.K., 115-168. http://dx.doi.org/10.4135/9781412973380.n5

Mangold, W. G.; Faulds, D. J. (2009). Social media: the new hybrid element of the promotion mix. Bus. Horizons, 52, 357-365. https://doi.org/10.1016/j.bushor.2009.03.002

Mercado, M.T. (2013). La especialización periodística como salida a la crisis de los medios. En Salas, M.I., Mira. E. (Coords.), Prospectivas y tendencias para la comunicación en el siglo XXI, 59-78. Madrid: CEU Ediciones.

Merchant, R.M., Elmer, S.; Lurie, N. (2011). Integrating Social Media into Emergency-Preparedness Efforts. New. Engl. J. Med., 365, 289-291. http://dx.doi.org/10.1056/nejmp1103591

Moorhead, S.A., Hazlett, D. E., Harrison, L., Carroll, J. K., Irwin, A. \& Hoving, C. (2013). A New Dimension of Health Care: Systematic Review of the Uses, Benefits, and Limitations of Social 
Media for Health Communication. Journal of Medical Internet Research, 15(4), e85. http://dx.doi.org/10.2196/jmir.1933

Neuendorf, K. (2017). The content analysis guidebook, 2nd ed.; SAGE Publications, Inc: Thousand Oaks, California.

Paulussen, S., \& Harder, R. A. (2014). Social Media References in Newspapers. Journalism Practice, 8(5), 542-551. http://dx.doi.org/10.1080/17512786.2014.894327

Păun, M. (2009). Perceptions on the Effectiveness of Communication between Public Institutions and Journalists through Social Media. Styles of Communication, 1, 121-140.

Piñuel Raigada, J.L. (2002). Epistemología, metodología y técnicas del análisis de contenido. Madrid: Universidad Complutense de Madrid.

Prasad, B. (2013). Social media, health care, and social networking. Gastrointest. Endosc., 77, 492495. http://dx.doi.org/10.1016/j.gie.2012.10.026

Rando-Cueto, D. \& de las Heras-Pedrosa, C. (2014). Presencia y estrategias de comunicación de hospitales andaluces en las redes sociales. Cuadernos Artesanos de Comunicación, 92, 155-173. http://dx.doi.org/10.4185/cac92

Rando-Cueto, D.; de las Heras-Pedrosa, C. (2016). Análisis de la comunicación corporativa de los hospitales andaluces vía twitter. Revista Opción, 32, 557-576. http://doi.org/10.5281/zenodo.3265951

Rando-Cueto, D.; Paniagua-Rojano, F.J. \& de las Heras-Pedrosa, C. (2016). Factores influyentes en el éxito de la comunicación hospitalaria vía redes sociales. Revista Latina de Comunicación Social, 71, 1.170 - 1.186. http://dx.doi.org/10.4185/rlcs-2016-1140

Riffe, D., Lacy, S. \& Fico, F. G. (2005). Analyzing media messages: Using quantitative content analysis in research. Mahwah. New Jersey. USA: Lawrence Erlbaum Associates.

Roman, L.A. (2014). Using Social Media to Enhance Career Development Opportunities for Health Promotion Professionals. Health Promotion Practice. 15, 471-475. http://dx.doi.org/10.1177/1524839914535213

Ruiz-Olabuénaga, J.I. (2012). Teoría y práctica de la investigación cualitativa. Bilbao: Deusto Digital

Russell, K. M.; Lamme, M. O. (2016). Theorizing public relations history: The roles of strategic intent and human agency. Public Relat. Rev., 42, 741-747. http://dx.doi.org/10.1016/j.pubrev.2016.04.002

Sánchez-Castillo, S. (2012). Representación social de las enfermedades raras en la prensa española. Aposta. Revista de ciencias sociales, 54(1). http://www.apostadigital.com/revistav3/hemeroteca/sscastillo.pdf 
Sánchez, M.T., Armayones, M.; Fernández-Luque, L., Gómez-Zúñigay, B. \& Pousada, M. (2012). Análisis del uso del video sanitario online en una muestra de redes sociales en España: implicaciones para la promoción de la salud. RevistaeSalud.com, 8(31), 7-13. https://dialnet.unirioja.es/servlet/articulo?codigo=4205991

Swan, M. (2009). Emerging Patient-Driven Health Care Models: An Examination of Health Social Networks, Consumer Personalized Medicine and Quantified Self-Tracking. International Journal of Environmental Research and Public Health, 6(2), 492-525. http://dx.doi.org/10.3390/ijerph6020492

Terrón Blanco, J.L. (2007). La comunicación para la salud en España, algunos apuntes. Revista ECO-Pos, 10(1), 23-44.

Tonia, T. (2014). Social media in public health: is it used and is it useful? Int. J. Public. Health, 59, 889-891. http://dx.doi.org/10.1007/s00038-014-0615-1

Vance, K.; Howe, W.; Dellavalle, R. P. (2009). Social internet sites as a source of public health information. Dermatol. Clin., 27, 133-136. https://doi.org/10.1016/j.det.2008.11.010

Van de Belt, T.H., Engelen, L.J., Berben, S.A., Teerenstra, S., Samsom, M., Schoonhoven, L. (2013). Internet and social media for health-related information and communication in health care: preferences of the Dutch general population. J. Med. Internet. Res., 15, e220. http://dx.doi.org/10.2196/jmir.2607

Ventola, C. L. (2014). Social media and health care professionals: benefits, risks, and best practices. $\begin{array}{llll}\text { Pharmacy and } & \text { Therapeutics, } & \text { 491-520. }\end{array}$ https://www.ncbi.nlm.nih.gov/pmc/articles/PMC4103576/

Zerfass, A.; Tench, R.; Verhoeven, P.; Verçid, D.; Moreno, A. European Communication Monitor 2018 (2018). Excellence in strategic communication-Key issues, leadership, gender and mobile media. Results of a survey in 42 countries. Brussels: EACD/EUPRERA, Quadriga Media Berlin: Berlin. http://www.zerfass.de/ECM-WEBSITE/ECM-2016.html

\section{AUTORES}

\section{Dolores Rando Cueto}

Doctoranda del Programa de Doctorado Interuniversitario de Comunicación de las Universidades de Cádiz, Huelva, Málaga y Sevilla. Licenciada en Periodismo y en Publicidad y Relaciones Públicas de la Universidad de Málaga. Participa en el desarrollo de una línea de investigación centrada en la comunicación que llevan a cabo instituciones sanitarias especializadas a través de las redes sociales y su efecto en la mejora de la calidad de vida de pacientes y familiares. La mayor parte de su trayectoria profesional la ha desempeñado en el ámbito hospitalario, en la Unidad de Comunicación del Hospital Regional Universitario de Málaga. Fruto de este trabajo, elaboró diferentes publicaciones encaminadas a la mejora de las labores comunicativas de la administración sanitaria. lrandocueto@uma.es

Orcid ID: https://orcid.org/0000-0002-8512-5034

GoogleScholar: https://scholar.google.com/citations?authuser=1\&user=2Zenw_8AAAAJ

Researcher ID: F-7686-2016 
Scopus Author ID: 57191969545

\section{Carlos de las Heras Pedrosa}

Profesor Titular de Universidad de Comunicación Audiovisual y Publicidad en la Universidad de Málaga, donde ha sido Vicerrector de Relaciones Institucionales y Gabinete del Rectorado, Director del Gabinete del Rectorado y Subdirector General de Comunicación. Actualmente codirector del Máster Universitario en Dirección Estratégica e Innovación en Comunicación. Su docencia e investigación se centra en la Comunicación Institucional y en la Comunicación Corporativa especialmente centrada en los sectores turísticos y de educación superior. Profesor investigador visitante en las universidades de Miami (USA), Sheffield, Cardiff y Leeds (UK). Profesor visitante en los programas de doctorado en las universidades de Guadalajara (Méjico) de Barinas, del Zulia y de Oriente (Venezuela).

cheras@uma.es

Orcid ID: https://orcid.org/0000-0002-2738-4177

GoogleScholar: https://scholar.google.es/citations?user=zsr7i8sAAAAJ

Researcher ID: M-4492-2015

Scopus Author ID: 57190015444

\section{Francisco Javier Paniagua Rojano}

Profesor Titular de Universidad. Licenciado (1996) y Doctor (2004) en Periodismo por la Universidad de Málaga. Ha sido profesor asociado (2003-2004), profesor colaborador (2007-2008) y contratado doctor (2008-2017) Actualmente es profesor de la Facultad de Ciencias de la Comunicación de la Universidad de Málaga, donde imparte la asignatura de Comunicación Institucional y Empresarial. Igualmente, desde 2008, es profesor invitado de los másteres en Comunicación Política e Institucional de la Fundación Ortega y Gasset y en Gestión Estratégica de la Comunicación e Innovación de la Universidad de Cádiz. También participa en la Escuela Internacional de Doctorado de la Universidad de Murcia, en el Doctorado en Ciencia Política y Administración Pública, en la línea de investigación Comunicación Política, Campañas y Elecciones y en los diferentes programas de doctorado en Comunicación en la Universidad de Málaga. Es secretario de la sección "Comunicación estratégica y organizacional" de la Asociación Española de la Investigación de la Comunicación (2007-actualidad). Hasta la fecha ha dirigido o co-dirigido nueve tesis doctorales sobre comunicación política, comunicación corporativa o empresa informativa; y más de 45 Trabajos Fin de Máster en la Universidad de Málaga y el Instituto Universitario Ortega y Gasset sobre los mismos temas y ha participado en dos proyectos de investigación del programa nacional -sobre credibilidad en los medios y social media y destinos turísticos-; en otros dos proyectos autonómicos sobre credibilidad de los medios en Andalucía. Entre marzo de 2011 y abril de 2014 compartió la docencia con la tarea de Director de Secretariado de Comunicación de la Universidad Internacional de Andalucía (UNIA), y ha sido durante varios años director de Comunicación de la Federación Andaluza de Municipios y Provincias (FAMP). Es revisor habitual de diferentes revistas de reconocido prestigio e impacto en el área.

fjpaniagua@uma.es

Orcid ID: http://orcid.org/0000-0001-7376-4536

GoogleScholar: https://scholar.google.es/citations?user=9z8ZuMMAAAAJ

Researcher ID: K-7709-2014

Scopus Author ID: 55579357500 